\title{
A Numerical Study of the Viscous Effects on the Particle Transportations due to a Solitary Wave Propagating over a Concavity
}

\author{
Chih-Hua Chang
}

\begin{abstract}
This study is focused on the interaction between a solitary wave and a bottom cavity with different aspect ratios. Vortex formations in cavity with different Reynolds numbers are considered. The moving trajectories of the primary vortex and the transportation of virtual fluid particles in the cavity are analyzed numerically. The numerical model is based on stream function-vorticity formulations, and the transient body-fitted grid combined with overset grid is adopted for grid systems. The numerical results were conducted through the comparisons with the experimental observations and measurements. Comparisons behave in good comparable tendency.
\end{abstract}

Index Terms-Solitary wave, cavity flow, viscous effect, particle motion.

\section{INTRODUCTION}

In the shallow water region, wave can easily transport particle materials, such as toxic pollutants, sand grains, containments, nutrition or planktons, etc., thereby influencing the ecological environment of the sea.

Almost all wave theories are treated by potential flow. However, vortex motions around the structure are occurred apparently. With the more complete considerations of real wave flow, in the past decade, several authors have investigated viscous effect in waves. The study of viscous effects on the propagation of solitary waves was initially investigated by [1]. Later, more and more numerical studies were presented in literatures (e.g. [2]-[5]). However, few researches outline the viscous effects of sediment transportation in coast environment. In this paper, the laminar viscous fluid flows are considered. Such as an oil tanker accident, causing the leakage of oil, large coastal region is polluted. Reynolds number $(R e)$ becomes an important role of parameter in viscous fluid motion.

This article is a part of the result of [6]. We apply the numerical model developed by [7]. The validation of the numerical model is conducted by comparisons of the particle tracking visualization in experiment [6]. The vortex behaviors of wave-cavity for simulation mainly at $R e=50000,5000$, and 500 are examined.

Manuscript received November 21, 2016; revised May 21, 2017. This work was supported by the project of Ministry of Scientific Technology, Taiwan, Republic of China (under MOST 104-2221-E-275-003)

Chih-Hua Chang is with the Ling-Tung University, No. 1, Ling-Tung Rd., Taichung, Taiwan, R.O.C. (e-mail: changbox@teamail.ltu.edu.tw).

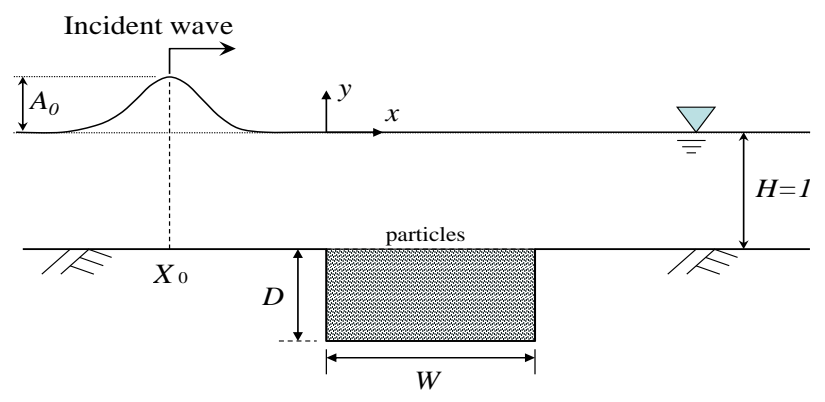

Fig. 1. Schematic diagram of physical problem.

\section{NUMERICAL METHOD}

\section{A. Flow-Field Equations}

A solitary wave propagating over a cavity in a two-dimensional, incompressible, viscous fluid flow is simulated (as illustrated in Fig. 1). All variables appeared in this paper are expressed in a non-dimensional form by using the normalized quantities defined by scales based on the undisturbed-water-depth, and the linear-long-wave speed. One may specify a stream function $(\psi)$ to satisfy the continuity equation. Let the components of particle velocity defined as $(u, v)=\left(\psi_{y},-\psi_{x}\right)$. Then substitute it into the definition of vorticity $(\omega)$. We can obtain the formulations of Poisson equation for stream function and the vorticity transport equation in a curvilinear coordinates. A transformation from Cartesian coordinates, $(x, y ; t)$, to a general boundary fitted-curvilinear $\operatorname{grid}_{(\xi, \eta ; \tau)}$. The $\psi-\omega$ formulation in general curvilinear coordinates reads

$$
\nabla^{2} \psi=-\omega
$$

$$
\frac{\nabla^{2} \omega}{R e}=\omega_{\tau}+U \omega_{\xi}+V \omega_{\eta}
$$

where

$$
\nabla^{2}=g^{11}\left(\frac{\partial^{2}}{\partial \xi^{2}}\right)+2 g^{12}\left(\frac{\partial}{\partial \xi}\right)\left(\frac{\partial}{\partial \eta}\right)+g^{22}\left(\frac{\partial^{2}}{\partial^{2} \eta}\right)+f^{1}\left(\frac{\partial}{\partial \xi}\right)+f^{2}\left(\frac{\partial}{\partial \eta}\right)
$$

$R e$ is Reynolds number. And the variables $U$ and $V$ are contravariant components of relative fluid velocity are given by

$U=\left(-x_{\tau} y_{\eta}+y_{\tau} x_{\eta}+\psi_{\eta}\right) / J ; V=\left(-y_{\tau} x_{\xi}+x_{\tau} y_{\xi}-\psi_{\xi}\right) / J$ 
In which

$$
\begin{gathered}
J=\left(x_{\xi} y_{\eta}-y_{\xi} x_{\eta}\right) \\
g^{11}=\left(x_{\eta}^{2}+y_{\eta}^{2}\right) / J^{2}, \\
g^{22}=\left(x_{\xi}^{2}+y_{\xi}^{2}\right) / J^{2}, \\
g^{12}=-\left(x_{\xi} x_{\eta}+y_{\xi} y_{\eta}\right) / J^{2}, \\
f^{1}=\left[\left(J g^{11}\right)_{\xi}+\left(J g^{12}\right)_{\eta}\right] / J, \text { and } \\
f^{2}=\left[\left(J g^{12}\right)_{\xi}+\left(J g^{22}\right)_{\eta}\right] / J .
\end{gathered}
$$

Equations (1) and (2) are to be solved with initial conditions and associated boundary conditions. The initial wave is imposed using the analytic solutions of [8] and is placed at $X_{0}$ in front of the cavity to keep away from disturbance. The initial conditions are a solitary wave of height $A_{0}$ on free surface. The celerity $(C)$ and initial wave $\operatorname{profile}\left(\zeta_{f}\right)$ are:

$$
C=1+\frac{1}{2} A_{0}-\frac{3}{20} A_{0}^{2}+\frac{3}{56} A_{0}^{3}
$$

$\zeta_{f}=A_{0} \cdot \sec h^{2} k\left[1-\frac{3}{4} A_{0} \cdot \tanh ^{2} k+A_{0}^{2}\left(\frac{5}{8} \tanh ^{2} k-\frac{101}{80} \sec h^{2} k \cdot \tanh ^{4} k\right)\right]$

In which, $k=\frac{\sqrt{A_{0}}}{2}\left(1-\frac{5}{8} A_{0}+\frac{71}{1280} A_{0}^{2}\right)\left(x-X_{0}\right)$. The free-surface boundary values for stream function can give as $\psi_{f}=C \zeta_{f}$. The vorticity values are all given to be zero at initial.

On the free surface, defined by $y=\zeta(x, t)$, the dynamic and kinematic boundary conditions must be satisfied. The dynamic condition is under the assumption of fluid pressure is the same as the atmospheric pressure. We neglect the surface tension on free surface. The total differential of atmosphere pressure $(\mathrm{Pa})$ along $\xi$-line is zero. This lead to

$$
\begin{aligned}
& \psi_{\xi \tau}\left(J g^{12}\right)+\psi_{\eta \tau}\left(J g^{22}\right)+\psi_{\xi}\left[-\left(\frac{x_{\eta}}{J}\right)_{t} x_{\xi}-\left(\frac{\zeta_{\eta}}{J}\right)_{t} \zeta_{\xi}\right] \\
& +\psi_{\eta}\left[\left(\frac{x_{\xi}}{J}\right)_{t} x_{\xi}+\left(\frac{\zeta_{\xi}}{J}\right)_{t} \zeta_{\xi}\right]+\left(u-x_{\tau}\right) u_{\xi} \\
& +\left(v-\zeta_{\tau}\right) v_{\xi}+\zeta_{\xi}+\omega\left[\left(u-x_{\tau}\right) \zeta_{\xi}-\left(v-\zeta_{\tau}\right) x_{\xi}\right] \\
& +\frac{J}{R e}\left(g^{12} \omega_{\xi}+g^{22} \omega_{\eta}\right)=0
\end{aligned}
$$

where $J=\left(x_{\xi} y_{\eta}-y_{\xi} x_{\eta}\right)$ is the Jacobian transformation. The kinematic condition on free surface is claimed that fluid particle which is once on the surface remains on it, and hence yields the condition

$$
\psi_{\xi}+\zeta_{\tau} x_{\xi}=x_{\tau} y_{\xi}
$$

The bottom is a constant, streamfunction can be written as $\psi_{0}=0$. And the vorticity on wall shall satisfy the velocity vanish at walls, a method derived by [9] but expressed in the present generalized cuevilinear form is given by

$$
\begin{aligned}
\omega_{0}= & 2 g^{22}\left(\psi_{0}-\psi_{1}+\psi_{0 \eta}\right) \\
& -\left(g^{11} \psi_{0 \xi \xi}+2 g^{12} \psi_{0 \xi \eta}+f^{1} \psi_{0 \xi}+f^{2} \psi_{0 \eta}\right)
\end{aligned}
$$

For the vertical or horizontal walls the (8) leads to

$$
\omega_{0}=-2 g^{i i}\left(\psi_{1}-\psi_{0}\right)
$$

where the grid node on wall is noted by subscript " 0 ", and " 1 " is the node adjacent to " 0 " one in interior flow field. The superscripts $i=1$ and $i=2$ are specified to vertial wall and horizontal bottom, respectively.

The open boundary conditions are given as

$$
\vartheta_{\tau} \pm \sqrt{1+\varsigma} \vartheta_{\xi} / x_{\xi}=0
$$

where $\vartheta$ is a dummy variables, representing $\psi, \omega$ or $\zeta$; the + sign is chosen for the right boundary; and the - sign is chosen for the left boundary.

\section{B. Numerical Method}

The streamfunction-vorticity with free surface (SVFS) formulation was first developed by [2]. They used boundary-fitted grid for solving the governing equations with Finite Analytic Method (FAM) [10] and discretizing the associate boundary conditions by time-averaged Finite Difference Method (FDM). Also, they joined the overset grid in SVFS model to reveal detail flow motions. The detail procedures in numerical method in grid generations and discretizations can refer these references. In this paper, we applied and modified their model. We use both of the FDM and FAM for suitable flow-field equations to increase computing efficiency. The base grids are built evenly in $x$-direction, while $y$-direction grid is separated into two parts. One part is distributed by a fixed even distribution in the lower part of water depth, another with the upper part to conform the moving free surface to re-distribute the $y$-grid space uniformly in this part. In the free surface, the free-surface displacements and stream functions are solved, respectively, from kinematic and dynamic conditions. A correction with time-average is a vital procedure when discrete the nonlinear free surface conditions. In flow field, we utilize the FAM discretization to solve both of the Poisson equations of stream function and the vorticity transport equation; meanwhile using the FDM to work out associated boundary conditions.

Unlike traditional FDMs, it is given by the local analytic solution to the linearized convective diffusion equation in a grid cell. This has the advantage of automatically weighting the convective diffusion. To express the two dimensional convective-diffusion equations in the standard FAM formulation, we let 


$$
\xi^{*}=\xi / \sqrt{g^{11}} ; \eta^{*}=\eta / \sqrt{g^{22}}
$$

Therefore (1) becomes

$2 A_{\psi} \psi_{\xi^{*}}+2 B_{\psi} \psi_{\eta^{*}}=\psi_{\xi^{*} \xi^{*}}+\psi_{\eta^{*} \eta^{*}}+S_{\psi}$

Where $A_{\psi}=-f^{1} / 2 / \sqrt{g^{11}} ; \quad B_{\psi}=-f^{2} / 2 / \sqrt{g^{22}} \quad, \quad$ and $S_{\psi}=-2 g^{12} \psi_{\xi \eta}-\omega$. The transformed transport equation for vorticity, (2), can be expressed as

$$
2 A_{\omega} \omega_{\xi^{*}}+2 B_{\omega} \omega_{\eta^{*}}=\omega_{\xi^{*} \xi^{*}}+\omega_{\eta^{*} \eta^{*}}+S_{\omega}
$$

where $A_{\omega}=\left(U \operatorname{Re}-f^{1}\right) / 2 / \sqrt{g^{11}} ; B_{\omega}=\left(V \operatorname{Re}-f^{2}\right) / 2 / \sqrt{g^{22}}$, and

$$
S_{\omega}=\left[2 g^{12} / \sqrt{g^{11} g^{22}}-\operatorname{Re}\left(\omega_{P}^{n+1}-\omega_{P}^{n}\right) / \Delta \tau\right] \omega_{\xi^{*} \eta^{*}}
$$

The cell spaces are $\Delta \xi^{*}=h=1 / \sqrt{g^{11}}$ and $\Delta \eta^{*}=k=1 / \sqrt{g^{22}}$. In the FAM, the governing differential equations are discretized into algebraic equations based on an analytic solution on the small computational element. In this method, a combined function of a linear and an exponential function for the boundary conditions is commonly used. The solutions of (12) and (13), expressed by the dummy variable $\varphi$ at node $P$ can be described with the nine-point values as

$$
\varphi_{p}=\sum_{i=N E}^{N C} C_{i} \varphi_{i}+S_{\varphi} C_{P}
$$

with the FAM coefficients given by

$$
\begin{gathered}
C_{E C}=E_{B} e^{-A h}, C_{W C}=E_{B} e^{A h}, C_{S C}=E_{A} e^{B k}, C_{N C}=E_{A} e^{-B k}, \\
C_{N E}=E e^{-A h-B k}, C_{N W}=E e^{A h-B k}, C_{S E}=E e^{-A h+B k}, C_{S W}=E e^{A h+B k}
\end{gathered}
$$

and

$$
\begin{aligned}
C_{p} & =\frac{1}{2\left(A^{2}+B^{2}\right)}\left[A h\left(C_{N W}+C_{W C}+C_{S W}-C_{N E}-C_{E C}-C_{S E}\right)\right. \\
& \left.+B k\left(C_{S W}+C_{S C}+C_{S E}-C_{N W}-C_{N C}-C_{N E}\right)\right]
\end{aligned}
$$

where

$$
\begin{gathered}
E=\frac{1}{4 \cosh (A h) \cosh (B k)}-A h E_{2} \operatorname{coth}(A h)-B k E_{2}^{\prime} \operatorname{coth}(B k), \\
E_{A}=2 A h \frac{\cosh ^{2} A h}{\sinh (A h)} E_{2}, \\
E_{B}=2 B h \frac{\cosh ^{2} B k}{\sinh (B k)} E_{2}^{\prime},
\end{gathered}
$$

$$
\begin{gathered}
E_{2}=\sum_{m=1}^{\infty} \frac{-(-1)^{m}\left(\lambda_{m} h\right)}{\left[(A h)^{2}+\left(\lambda_{m} h\right)^{2}\right]^{2} \cosh \left(\mu_{m} k\right)}, \\
E_{2}^{\prime}=\sum_{m=1}^{\infty} \frac{-(-1)^{m}\left(\lambda_{m}^{\prime} k\right)}{\left[(B k)^{2}+\left(\lambda_{m}^{\prime} k\right)^{2}\right]^{2} \cosh \left(\mu_{m}^{\prime} h\right)}, \\
\mu_{m}=\sqrt{A^{2}+B^{2}+\lambda_{m}^{2}}, \\
\lambda_{m}=(2 m-1) \pi / 2 / h, \\
\mu_{m}^{\prime}=\sqrt{A^{2}+B^{2}+\left(\lambda_{m}^{\prime}\right)^{2}}, \text { and } \\
\lambda_{m}^{\prime}=(2 m-1) \pi / 2 / k .
\end{gathered}
$$

Above, the $A$ and $B$ are replaced by $A_{\psi}$ and $B_{\psi}$ for solving (1); and by $A_{\omega}$ and $B_{\omega}$ for solving (2).

\section{RESUlts}

\section{A. Visualization Comparisons}

Fig. 2 compares the results of experimental visualization and numerical simulation, thereby verifying the consistency of both. The $R e$ is approximately equal to 66700 according to the experimental conditions. Incident wave with $A_{0}=0.2$ is placed at $X_{0}=-20$ to keep away from the cavity. The streamline patterns of a solitary wave interacting with a square cavity with $D=W=0.43$ at various instant snapshots are plotted in Fig. 2a, while the corresponding image of Fig. $2 \mathrm{~b}$ taken by PIV technique to capture the velocity vectors; and the snapshot of particle trajectory photography at $t=33$ are shown in Fig. 2c. It shows the main wave moves to downstream, only local flow evolves around the cavity, the primary vortex moves back toward the center of cavity; simutaneously, all eddies are swelling, the secondary vortex is bulging obviously at the cavity right corner. Results show the corresponding observed flow visualizations, basically have the same characteristics to numerical solutions.

For a different aspect ratio with $W=0.215$, and $D=0.43$, which is half of the previous case in cavity width. Similarly, the computational streamlines at one instant moment $(t=35)$ are compared with photo pictures. The comparisons between the numerical streamline patterns, velocity vectors and the experimental photoes are in good agreements, as shown in Fig. 3. In comparison with the previous case, the major different behavior is the secondary vortex in the cavity right wall can move toward the deeper cavity. But the fluid is almost stationary below the lower half of this deep cavity. This means the contents deposited in cavity deeper than the half-water depth will not be easily disturbed by a moderate solitary wave. Above results can validate the precision of present model to build confidence to calculate the following numerical examples. 


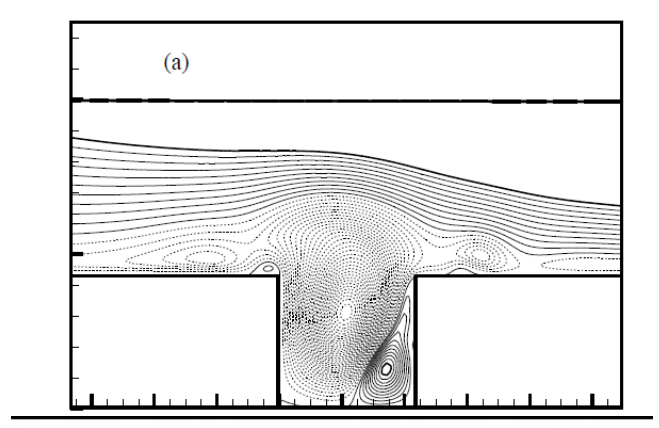

(b)

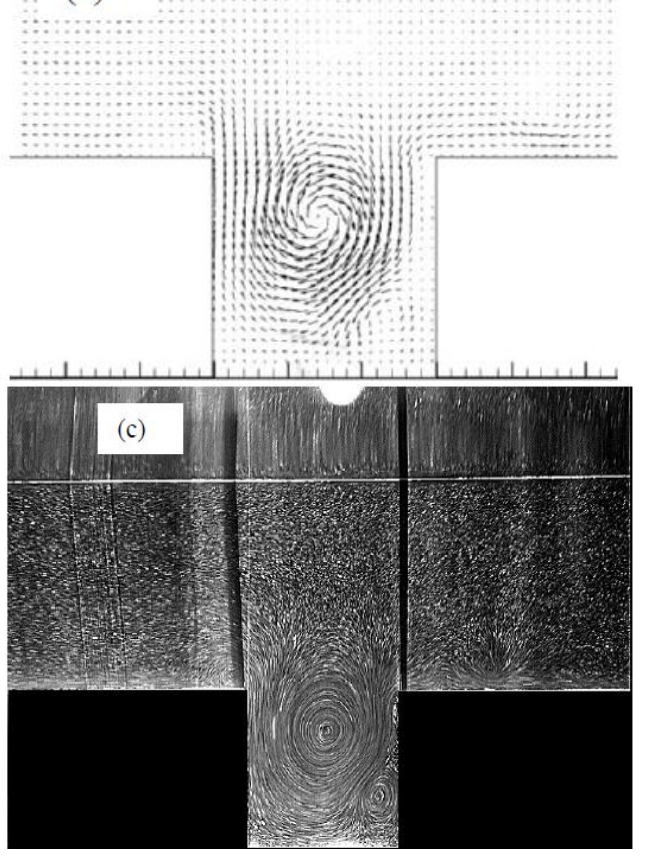

Fig. 2. Solitary wave $A_{0}=0.2$ passes a cavity of $W=D=0.43$ : (a) numerical streamlines $(-0.011 \sim 0.002$ at interval 0.0002 with light lines, solid line is positive and dashed line is negative; $0.002 \sim 0.2$ at interval 0.005 with heavy solid lines); (b) PIV measurement of velocity vectors, and (c) flow visualization pictures by experiment [6].

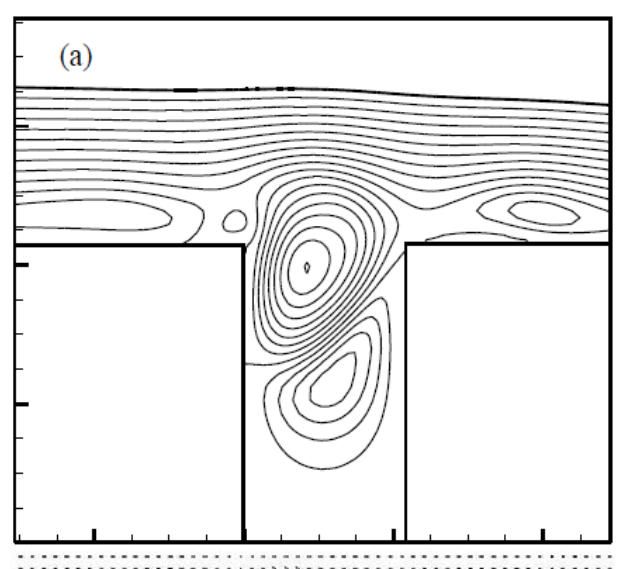

(b)

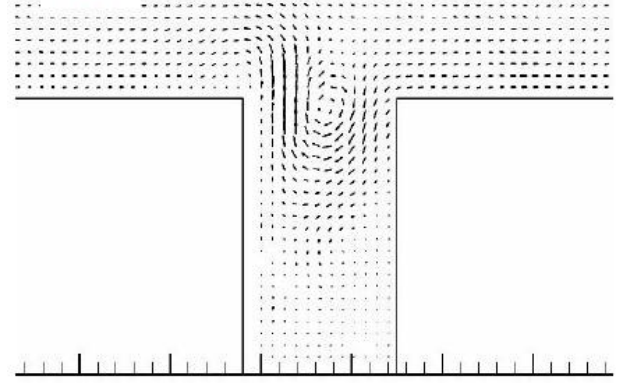

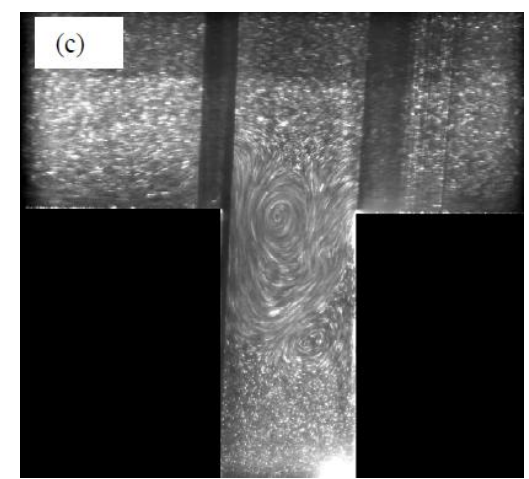

Fig. 3. Solitary wave $A_{0}=0.2$ passes a cavity of $W=0.215$ and $D=0.43$ : (a) numerical streamlines $(-0.005 \sim 0.002$ at interval 0.0002 with light lines; 0.002 0.2 at interval 0.005 with heavy solid lines); (b) PIV measurement of velocity vectors, and (c) flow visualization pictures by experiment [6].

(a) $R e=50000$
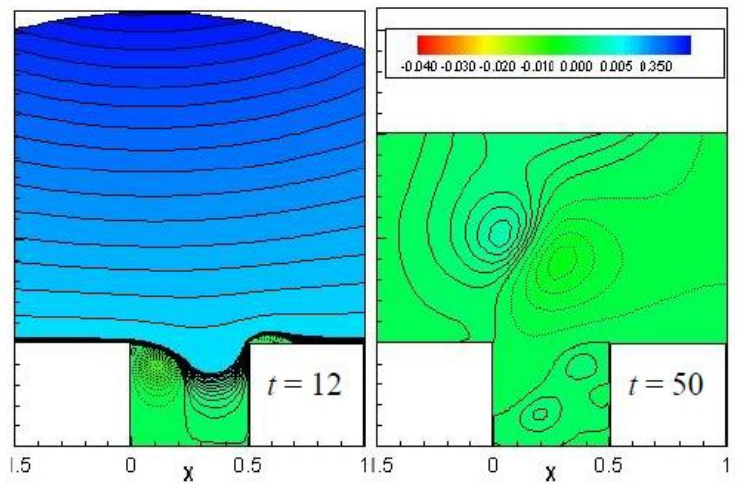

(a) $R e=50000$
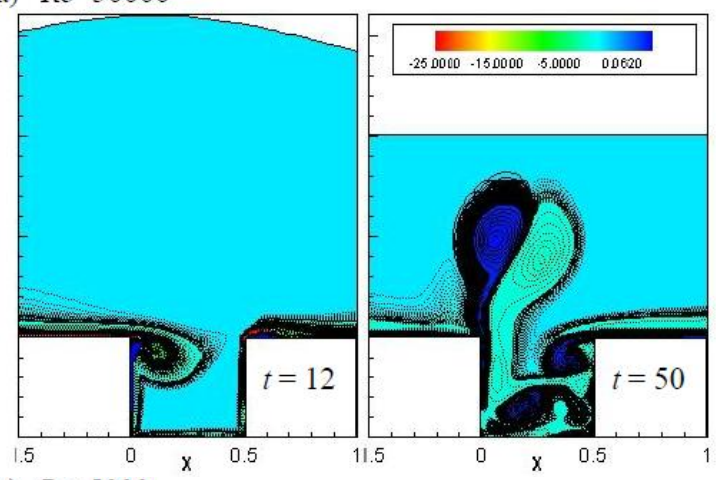

(b) $R e=5000$

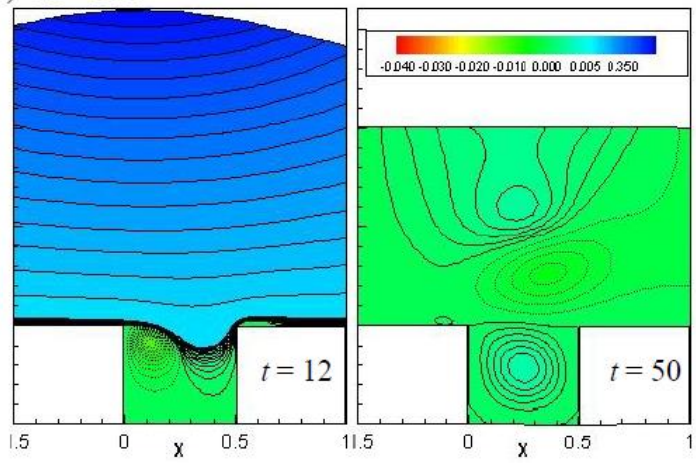

Fig. 4. Streamlines of a solitary wave $A 0=0.6$ passes a cavity of $W=D=0.5$ for different $R e$ at various time: (a) $R e=50000$; (b) $R e=5000$; (c) $R e=500$.

\section{B. Flow Patterns at $R e=50000,5000$, and 500}

It can easily be specified a pure bottom cavity in numerical simulations. The purpose of this section is to discuss the role of fluid viscosity, this includes $R e=50000,5000$, and 500. A pure cavity with $W=D=0.5$ in geometry model is selected. A 
solitary wave passing over it with $A_{0}=0.6$ starting from $X_{0}=$ -15 is concerned. Relatively, that is a case of steep wave. Fig. 4 shows two snap shots of streamlines. Contours are plotted with $-0.04 \sim 0$ at interval $0.001 ; 0 \sim 0.0065$ at interval 0.0005 and $0.05 \sim 0.75$ at interval 0.05 . The $R e=50000$ (Fig. 4a) is a case closer to general laboratory water wave conditions. The vortex is shedding out when the wave passes over the cavity. There is a clockwise vortex, which is the primary one generated at the edge due to flow separation $(t=12)$. The flow separation is due to high velocity gradient of boundary layer of incident solitary wave near the frontal edge of the cavity. In the same time, a vortex is generated at the top of the downstream bottom. Actually, it is relatively small but the numbers of vortices increase with time. The primary vortex grows larger and rotates continuously, and size of the vortex increases simultaneously. At the diffusion stage $(t=50)$, the flow motion is gradually weaker and weaker. The secondary vortex generated from the concave right corner develops into a closed cavity flow. A main anti-clockwise vortex rotates forming concentric streamlines in the cavity. The jetting pair vortices is not found for $R e=500$ (Fig. 4c). In addition, the closed cavity flow, acts like the case of $R e=5000$, but has weaker flow motion in it. Fig. 5 shows the equivalent vorticity line corresponding to the same instance of Fig. 4. Contours $-0.04 \sim 0.686$ at interval $0.002 ;-25 \sim-0.1$ at interval 0.1 are plotted with dashed lines for negative values. From Fig. 5, we observe that as the $R e$ increases the vorticity contours indicate that strong vorticity gradients develop on the bottom and the cavity walls. The pair of vorticity rolls move upward due to the stronger convection effect; for lower $R e(R e=500)$ the vorticity is distributed a symmetric pattern when it develops into a diffusion stage, that is similar to the lid-cavity flow behavior at very low $\operatorname{Re}[11]$.

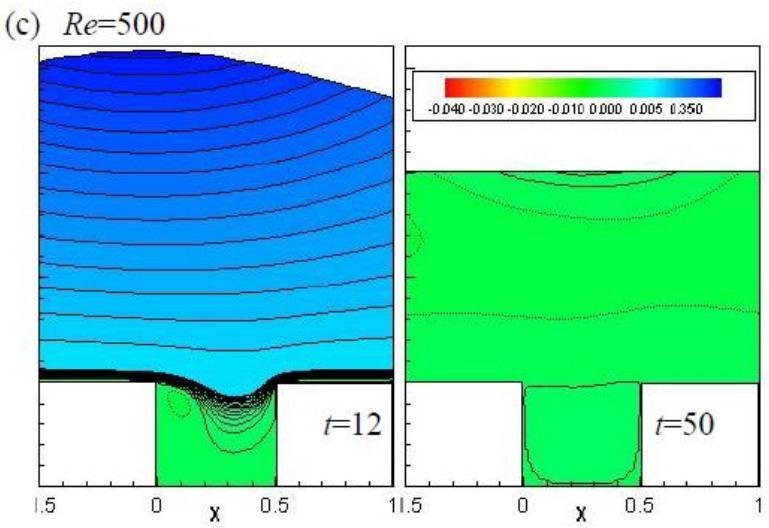

(b) $R e=5000$

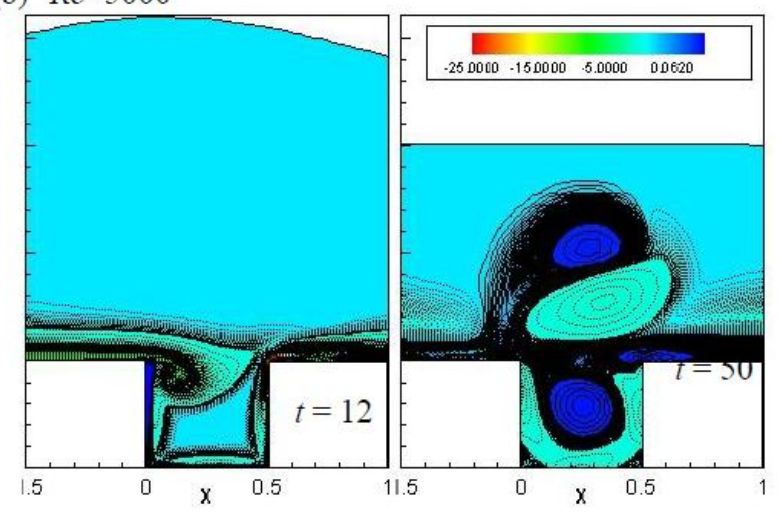

(c) $R e=500$

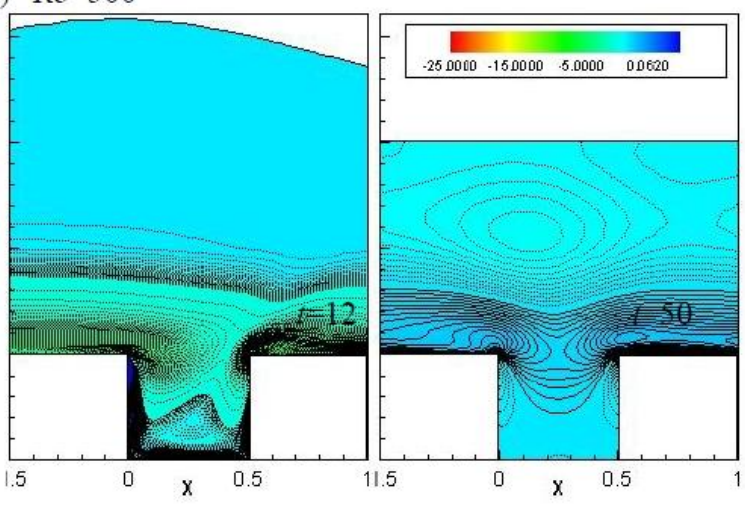

Fig. 5. Equi-vorticity contours of a solitary wave $A_{0}=0.6$ passes a cavity of $W$ $=D=0.5$ for different Re at various time: (a) $R e=50000$; (b) $R e=5000$; (c) $R e=500$

(a) $R e=50000$
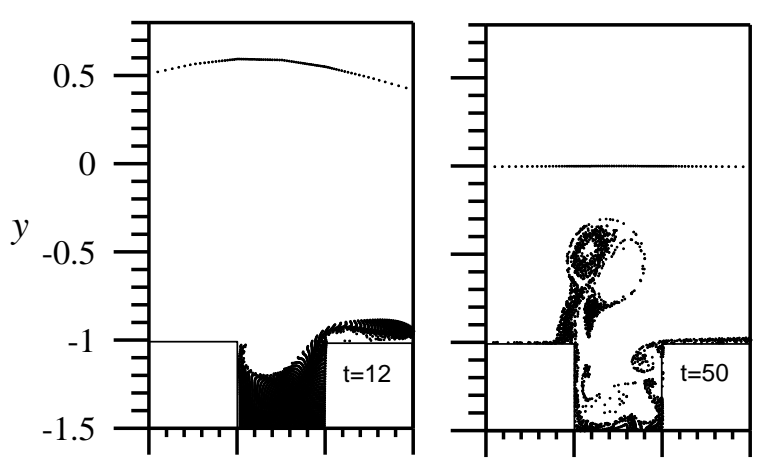

(b) $R e=5000$
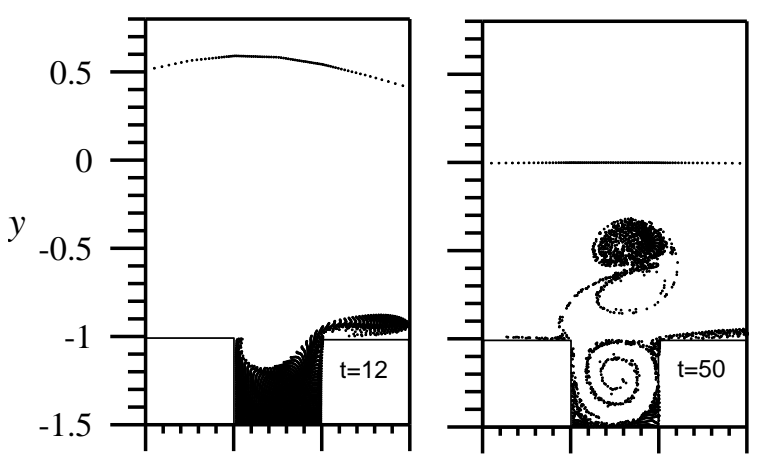

(c) $R e=500$
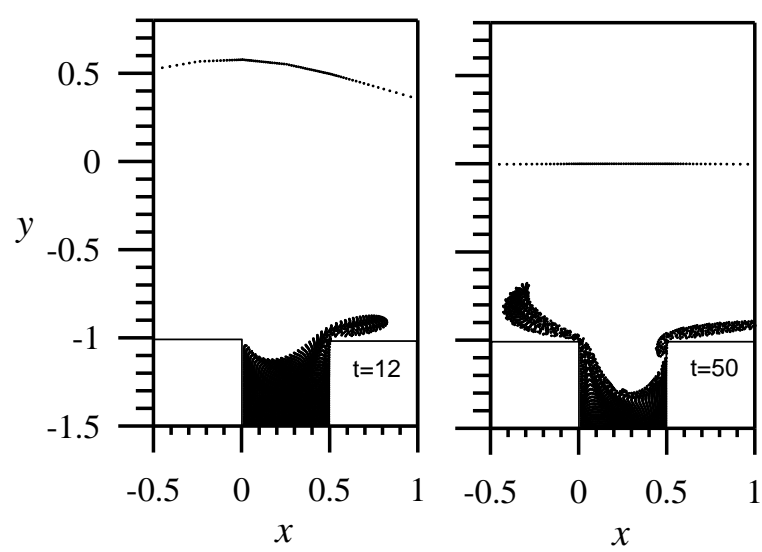

Fig. 6. Time varying particle motion for $A_{0}=0.6$ passing over a square cavity at different $R e$. 


\section{Particle Motions in Cavity}

The particles within the cavity can be partially transported outside when wave passes over. Fig. 6 shows the migration of particles resulting from the interaction of the solitary wave of $A_{0}=0.6$ and a cavity with $W=D=0.5$ at $R e=50000,5000$, and 500. We select the spaces $\delta x=\delta y=0.01$ to determine the particle density in the cavity. There are 2500 imagined fluid particles within this cavity. The particle motions are traced using the Lagrangian viewpoint. The phenomenon of particle motions at $t=12$, and 50 are plotted. Based on the figures we inspect that the role of $R e$ in the particle transportations. For the case of $R e=50000$, from Fig. 6a we can find when the wave is approaching cavity, at $t=12$ partial particles have been carried downstream. Finally, the left wall of cavity is suffered a vertical velocity shooting upwards, thereby producing a jet-like flow along the wall, and the jet splits into bidirectional asymmetric particle rolls. The left roll of the pair usually carries more particles than the right one. The rolling mechanisms for $R e=50000$ (Fig. 6a) and $R e=5000$ (Fig. 6b) perform in a similar pattern. Major differences in $R e=5000$ are the pair rolls growing upward and tilted toward downstream and a system of spiral distribution is formed in cavity at the final stage. For lower Reynolds number, $R e=500$ particles move slowly without apparent particle rolls produced during the wave propagation process, but a group of particles are swept upstream. Anyway, once the particles brought outside by flow motion, if there are currents or waves will carry these particles to other region, thereby affecting the environment.

\section{CONCLUSIONS}

This paper introduces the numerical and experimental methods to analyze the motion of a solitary wave stirring the particles in a cavity. The numerical model is governed by streamfunction-vorticity equations using a body-fitted grid system and suitable FAM to obtain converged flow field solutions. The emphasis of this research is to study the mechanism of viscous effect in the translation of vortex motion and the transportation of virtual particles around a cavity. As we think, a serious impact on the environment occurred when an additional disturbance, such as waves or currents, transporting these particles to drift into downstream. Some important items are noted here as conclusions.

The comparison between the numerical simulations and experimental visualizations for the vortex motion due to wave-cavity interaction are in good agreement for two different aspect ratio cavities.

The flow cases for $R e=50000,5000$, and 500 are simulated in numerical study. For the low $R e$ flow, the evolving vortex motion in cavity behaves like the traditional lid-cavity flow, and no vortices produced by uplifting jet flow. The path of primary vortex core moves more smooth and gentle, at the final stage, it moving inclines into upper left; that is in contrast to the higher $R e$, which tends towards upper right.

\section{ACKNOWLEDGMENT}

This study has been sponsored by the project of Ministry of Scientific Technology, Taiwan, Republic of China (Serial No. MOST 104-2221-E-275-003).

\section{REFERENCES}

[1] C. J. Tang, V. C. Patel, and L. Landweber, "Viscous effects on propagation and reflection of solitary waves in shallow channel," $J$ Computational Physics, vol. 88, pp. 86-113, 1990.

[2] C. J. Tang and J. H. Chang, "Flow separation during a solitary wave passing over a submerged obstacle," J Hydraul Eng, vol. 124, no. 7, pp. 732-749, 1998.

[3] C. M. Dong and C. J. Huang, "Generation and propagation of water waves in a two-dimensional numerical viscous wave flume," Journal of Waterway, Port, Coastal, and Ocean Engineering, vol. 130, no. 3 , pp. 143-153, 2004

[4] D. C. Lo and D. L. Young, "Numerical simulation of solitary waves using velocity-vorticity formulation of Navier-Stokes equations," Journal of Engineering Mechanics, vol. 132, no. 2, pp. 211-219, 2006.

[5] P. L. F. Liu and K. Al-Banaa, "Solitary wave run-up and force on a vertical barrier," J Fluid Mech, vol. 505, pp. 225-233, 2004.

[6] C. H. Chang and C. Lin, "Effect of solitary wave on viscous-fluid flow in bottom cavity," Environ Fluid Mech, vol. 15, pp. 1135-1161, 2015

[7] C. H. Chang, C. J. Tang, and C. Lin, "Vortex generation and flow pattern development after a solitary wave passing over a bottom cavity," Computers and Fluids, vol. 53, pp. 79-92, 2012.

[8] R. Grimshaw, "The solitary wave in water of variable depth (Part 2)," $J$ Fluid Mech, vol. 46, part 3, pp. 611-622, 1971.

[9] M. Nallasamy, "Numerical solution of the separation flow due to an obstruction," Computers \& Fluids, vol. 14, no. 1, pp. 59-68, 1986.

[10] C. J. Chen and H. C. Chen, "Finite analytic numerical method for unsteady two-dimensional Navier Stokes equations," J Computational Physics, vol. 53, pp. 209-226, 1984

[11] Z. F. Tian and P. X. Yu, "An efficient compact difference scheme for solving the streamfunction formulation of the incompressible Navier-Stokes equations," J of Computational Physics, vol. 230, pp. 6404-6419, 2011.

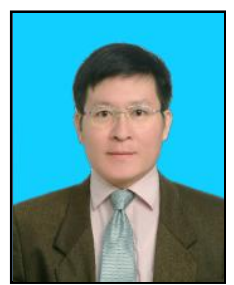

Chih-Hua Chang is an associate professor of the Department of Information Management, and the Natural Science Division in General Education Center at Ling-Tung University in Taiwan. He earned a Ph.D. degree in 1997 in department of hydraulic and ocean engineering of National Cheng Kung University, Taiwan. He has been at Ling-Tung University since 2003. Dr. Chang's research interests include geographic information system, coastal and ocean engineering, and computational fluid dynamics. During his research processes, he has been personally developed models including 1D Boussinesq adaptive grid model, 2D (quasi-3D) shallow-water wave model with bottom curvature, Vertical 2D-Streamfunction model, Vertical 2D-Streamfunction-vorticity internal flow model, Vertical 2D-Streamfunction-vorticity external flow (free surface) model, and 3D fully-nonlinear potential-flow wave model. 